\title{
GENERALIZED CLASS OF UNIVALENT FUNCTIONS WITH TWO FIXED POINTS
}

\author{
B. A. URALEGADDI AND C. SOMANATHA
}

\begin{abstract}
Univalent functions of the form $f(z)=a_{1} z-\sum_{n=2}^{\infty} a_{n} z^{n}$, where $a_{n} \geq 0$, are dealt with. We examine the subclasses for which $(1-\lambda) f\left(z_{0}\right) / z_{0}+\lambda f^{\prime}\left(z_{0}\right)=1\left(-1<z_{0}<1\right)$. The coefficient inequalities and the extreme points of the classes that are starlike and convex of order $\alpha$ are determined. Many of the results of Silverman are obtained as particular cases.
\end{abstract}

\section{Introduction}

In [1], Silverman examined the class of functions of the form

$$
f(z)=z-\sum_{n=2}^{\infty}\left|a_{n}\right| z^{n}
$$

that are univalent in the unit disk $\Delta=\{z:|z|<1\}$. In [2], silverman studied the class of univalent functions of the form

$$
f(z)=a_{1} z-\sum_{n=2}^{\infty} a_{n} z^{n}, \quad a_{n} \geq 0
$$

where either

$$
f\left(z_{0}\right)=z_{0}, \quad\left(-1<z_{0}<1 ; z_{0} \neq 0\right)
$$

or

Received Decemeber 16, 1991.

1980 Mathematics subject classifications (1985 revision). Primary $30 \mathrm{C} 45$.

Key words and phrases: Univalent functions, starlike, convex, extreme points. 


$$
f^{\prime}\left(z_{0}\right)=1 \quad\left(-1<z_{0}<1\right) .
$$

In this paper we consider functions of the form

$$
f(z)=a_{1} z-\sum_{n=2}^{\infty} a_{n} z^{n}
$$

where

$$
a_{n} \geq 0,(1-\lambda) f\left(z_{0}\right) / z_{0}+\lambda f^{\prime}\left(z_{0}\right)=1 \quad\left(-1<z_{0}<1 ; 0 \leq \lambda \leq 1\right) .
$$

A function $f(z)$ is said to be starlike of order $\alpha, 0 \leq \alpha<1$, if

$$
\operatorname{Re}\left\{z f^{\prime}(z) / f(z)\right\}>\alpha \quad(|z|<1)
$$

and is said to be convex of order $\alpha$ if

$$
\operatorname{Re}\left\{1+z f^{\prime \prime}(z) / f^{\prime}(z)\right\}>\alpha \quad(|z|<1) .
$$

Given $\alpha$ and $z_{0}$ fixed let $S_{\lambda}^{*}\left(\alpha, z_{0}\right)$ and $K_{\lambda}\left(\alpha, z_{0}\right)$ be the subclasses of functions starlike of order $\alpha$ and convex of order $\alpha$ respectively satisfying (1). For these classes we obtain coefficient estimates, comparable results and extreme points.

The special cases of $S_{\lambda}^{*}\left(\alpha, z_{0}\right)$ and $K_{\lambda}\left(\alpha, z_{0}\right)$ are the subclasses $S_{0}^{*}\left(\alpha, z_{0}\right)$, $S_{1}^{*}\left(\alpha, z_{0}\right), K_{0}\left(\alpha, z_{0}\right)$ and $K_{1}\left(\alpha, z_{0}\right)$ respectively. Several results on these subclasses may be found in [2].

We need the following results due to Silverman [2].

Theorem A. A function $f(z)=a_{1} z-\sum_{n=2}^{\infty} a_{n} z^{n}\left(a_{n} \geq 0\right)$ is starlike of order $\alpha$ if and only if $\sum_{n=2}^{\infty}(n-\alpha) a_{n} \leq a_{1}(1-\alpha)$.

Theorem B.A function $f(z)=a_{1} z-\sum_{n=2}^{\infty} a_{n} z^{n}\left(a_{n} \geq 0\right)$ is convex of order $\alpha$ if and only if $\sum_{n=2}^{\infty} n(n-\alpha) a_{n} \leq a_{1}(1-\alpha)$. 


\section{Coefficient Inequalities}

Theorem 1. Suppose $a_{n} \geq 0$ for every $n$. Then $f(z)=a_{1} z-\sum_{n=2}^{\infty} a_{n} z^{n}$ is in $S_{\lambda}^{*}\left(\alpha, z_{0}\right)$ if and only if $\sum_{n=2}^{\infty} a_{n}\left[\frac{(n-\alpha)}{(1-\alpha)}-((1-\lambda)+n \lambda) z_{0}^{n-1}\right] \leq 1$.

Proof. Since

$$
\begin{aligned}
& (1-\lambda) f\left(z_{0}\right) / z_{0}+\lambda f^{\prime}\left(z_{0}\right) \\
= & (1-\lambda)\left(a_{1}-\sum_{n=2}^{\infty} a_{n} z_{0}^{n-1}\right)+\lambda\left(a_{1}-\sum_{n=2}^{\infty} n a_{n} z_{0}^{n-1}\right) \\
= & a_{1}-\sum_{n=2}^{\infty} a_{n}[(1-\lambda)+n \lambda] z_{0}^{n-1}=1,
\end{aligned}
$$

we have

$$
a_{1}=1+\sum_{n=2}^{\infty} a_{n}[(1-\lambda)+n \lambda] z_{0}^{n-1} .
$$

Substituting this value of $a_{1}$ in the statement of Theorem $A$ we get

$$
\sum_{n=2}^{\infty} a_{n}\left[\frac{(n-\alpha)}{(1-\alpha)}-((1-\lambda)+n \lambda) z_{0}^{n-1}\right] \leq 1
$$

Corollary 1. If $f(z)=a_{1} z-\sum_{n=2}^{\infty} a_{n} z^{n}$ is in $S_{\lambda}^{*}\left(\alpha, z_{0}\right)$ then

$$
a_{n} \leq(1-\alpha) /\left[(n-\alpha)-(1-\alpha)((1-\lambda)+n \lambda) z_{0}^{n-1}\right] \quad(n=2,3, \ldots)
$$

with equality for

$$
f(z)=\frac{(n-\alpha) z-(1-\alpha) z^{n}}{(n-\alpha)-(1-\alpha)((1-\lambda)+n \lambda) z_{0}^{n-1}} .
$$

Theorem 2. Let $f(z)=a_{1} z-\sum_{n=2}^{\infty} a_{n} z^{n}\left(a_{n} \geq 0\right)$ and satisfy (1). Then $f(z)$ is univalent if and only if $\sum_{n=2}^{\infty} a_{n}\left[n-((1-\lambda)+n \lambda) z_{0}^{n-1}\right] \leq 1$. 
Proof. Since $a_{1}=1+\sum_{n=2}^{\infty} a_{n}[(1-\lambda)+n \lambda] z_{0}^{n-1}$, it suffices to show that $f(z)$ is univalent if and only if $\sum_{n=2}^{\infty} n a_{n} \leq a_{1}$.

Suppose $\sum_{n=2}^{\infty} n a_{n}>a_{1}$. We can write $\sum_{n=2}^{\infty} n a_{n}=a_{1}+\varepsilon,(\varepsilon>0)$. Then there exists an integer $N$ such that

$$
\sum_{n=2}^{N} n a_{n}>a_{1}+\frac{\varepsilon}{2} .
$$

For $z$ in the interval $\left(a_{1} /\left(a_{1}+\frac{\varepsilon}{2}\right)\right)^{\frac{1}{(N-1)}}<z<1$, we have

$$
\begin{aligned}
f^{\prime}(z) & \leq a_{1}-\sum_{n=2}^{N} n a_{n} z^{n-1} \\
& \leq a_{1}-z^{N-1} \sum_{n=2}^{N} n a_{n} \\
& <a_{1}-z^{N-1}\left(a_{1}+\frac{\varepsilon}{2}\right)<0 .
\end{aligned}
$$

Since $f^{\prime}(0)>0$, there exists a real number $z_{0}, 0<z_{0}<1$, such that $f^{\prime}\left(z_{0}\right)=0$. Hence $f$ is not univalent.

Conversely, let $\sum_{n=2}^{\infty} a_{n}\left[n-((1-\lambda)+n \lambda) z_{0}^{n-1}\right] \leq 1$. Then by Theorem 1 , $f \in S_{\lambda}^{*}\left(0, z_{0}\right)$. Hence $f$ is univalent.

Theorem 3. Suppose $a_{n} \geq 0$ for every $n$. Then $f(z)=a_{1} z-\sum_{n=2}^{\infty} a_{n} z^{n}$ is in $K_{\lambda}\left(\alpha, z_{0}\right)$ if and only if $\sum_{n=2}^{\infty} a_{n}\left[\frac{n(n-\alpha)}{(1-\alpha)}-((1-\lambda)+n \lambda) z_{0}^{n-1}\right] \leq 1$.

Proof. Substituting the value of $a_{1}$ given by (2), in the statement of Theorem $B$ the result follows.

Corollary 2. If $f(z)=a_{1} z-\sum_{n=2}^{\infty} a_{n} z^{n}$ is in $K_{\lambda}\left(\alpha, z_{0}\right)$ then

$$
a_{n} \leq(1-\alpha) /\left[n(n-\alpha)-(1-\alpha)((1-\lambda)+n \lambda) z_{0}^{n-1}\right] \quad(n=2,3, \ldots)
$$


with equality for

$$
f(z)=\frac{n(n-\alpha) z-(1-\alpha) z^{n}}{n(n-\alpha)-(1-\alpha)((1-\lambda)+n \lambda) z_{0}^{n-1}}
$$

\section{Comparable Results}

Theorem 4. If $f(z) \in K_{\lambda}\left(\alpha, z_{0}\right)$ then $f(z) \in S_{\lambda}^{*}\left(2 /(3-\alpha), z_{0}\right)$.

Proof is similar to that of Theorem 3 in [2]. The result is sharp, with extremal function

$$
f(z)=\frac{2(2-\alpha) z-(1-\alpha) z^{2}}{2(2-\alpha)-(1-\alpha)((1-\lambda)+2 \lambda) z_{0}}
$$

Theorem 5. If $f(z) \in S_{\lambda}^{*}\left(\alpha, z_{0}\right)$ then $f(z)$ is convex in the disk

$$
|z|<r=r(\alpha)=\inf _{n}\left[\frac{n-\alpha}{n^{2}(1-\alpha)}\right]^{\frac{1}{n-1}} \quad n=2,3, \ldots
$$

The result is sharp for the extremal function

$$
f_{n}(z)=\frac{(n-\alpha) z-(1-\alpha) z^{n}}{(n-\alpha)-(1-\alpha)((1-\lambda)+n \lambda) z_{0}^{n-1}} \quad(n=2,3, \ldots) .
$$

Proof. It suffices to show that

$$
\left|z \frac{f^{\prime \prime}(z)}{f^{\prime}(z)}\right| \leq 1 \quad \text { for } \quad|z|<r(\alpha) .
$$

We have

$$
\left|z \frac{f^{\prime \prime}(z)}{f^{\prime}(z)}\right| \leq \frac{\sum_{n=2}^{\infty} n(n-1) a_{n}|z|^{n-1}}{a_{1}-\sum_{n=2}^{\infty} n a_{n}|z|^{n-1}}
$$

Thus

$$
\left|z \frac{f^{\prime \prime}(z)}{f^{\prime}(z)}\right| \leq 1 \text { if }
$$




$$
\begin{aligned}
& \sum_{n=2}^{\infty} n(n-1) a_{n}|z|^{n-1} \leq a_{1}-\sum_{n=2}^{\infty} n a_{n}|z|^{n-1} \\
= & 1+\sum_{n=2}^{\infty} a_{n}((1-\lambda)+n \lambda) z_{0}^{n-1}-\sum_{n=2}^{\infty} n a_{n}|z|^{n-1}
\end{aligned}
$$

that is if

$$
\sum_{n=2}^{\infty} a_{n}\left[n^{2}|z|^{n-1}-((1-\lambda)+n \lambda) z_{0}^{n-1}\right] \leq 1
$$

From Theorem 1, we have

$$
\sum_{n=2}^{\infty} a_{n}\left[\frac{(n-\alpha)}{(1-\alpha)}-((1-\lambda)+n \lambda) z_{0}^{n-1}\right] \leq 1
$$

Hence (3) will be true if

$$
n^{2}|z|^{n-1}-((1-\lambda)+n \lambda) z_{0}^{n-1} \leq \frac{(n-\alpha)}{(1-\alpha)}-((1-\lambda)+n \lambda) z_{0}^{n-1}
$$

solving (4) for $|z|$ we get

$$
|z| \leq\left[\frac{n-\alpha}{n^{2}(1-\alpha)}\right]^{\frac{1}{n-1}} \quad n=2,3, \ldots
$$

the result follows.

Remark. The conclusions in Theorem 4 and 5 are independent of $\lambda$ and the fixed point $z_{0}$.

\section{Extreme Points}

Theorem 6. The extreme points of $S_{\lambda}^{*}\left(\alpha, z_{0}\right)$ are given by $f_{1}(z)=z$ and $f_{n}(z)=\frac{(n-\alpha) z-(1-\alpha) z^{n}}{(n-\alpha)-(1-\alpha)((1-\lambda)+n \lambda) z_{0}^{n-1}}(n=2,3, \ldots)$.

Proof. It suffices to show that $f(z) \in S_{\lambda}^{*}\left(\alpha, z_{0}\right)$ if and only if it can be expressed in the form $f(z)=\sum_{n=1}^{\infty} \lambda_{n} f_{n}(z)$ where $\lambda_{n} \geq 0$ and $\sum_{n=1}^{\infty} \lambda_{n}=1$. 
Suppose $f(z)=\sum_{n=1}^{\infty} \lambda_{n} f_{n}(z)$, where $\lambda_{n} \geq 0$ and $\sum_{n=1}^{\infty} \lambda_{n}=1$. Then

$$
\begin{aligned}
f(z)= & \lambda_{1} f_{1}(z)+\sum_{n=2}^{\infty} \lambda_{n} f_{n}(z) \\
= & {\left[\lambda_{1}+\sum_{n=2}^{\infty} \frac{\lambda_{n}(n-\alpha)}{(n-\alpha)-(1-\alpha)((1-\lambda)+n \lambda) z_{0}^{n-1}}\right] z } \\
& -\sum_{n=2}^{\infty} \frac{\lambda_{n}(1-\alpha) z^{n}}{(n-\alpha)-(1-\alpha)((1-\lambda)+n \lambda) z_{0}^{n-1}}
\end{aligned}
$$

Note that

$$
\begin{aligned}
& {\left[(1-\lambda) f\left(z_{0}\right) / z_{0}\right]+\lambda f^{\prime}\left(z_{0}\right) } \\
= & {\left[(1-\lambda) \lambda_{1} f_{1}\left(z_{0}\right) / z_{0}\right]+(1-\lambda) \sum_{n=2}^{\infty} \lambda_{n} f_{n}\left(z_{0}\right) / z_{0} } \\
& +\lambda \lambda_{1} f_{1}^{\prime}\left(z_{0}\right)+\lambda \sum_{n=2}^{\infty} \lambda_{n} f_{n}^{\prime}\left(z_{0}\right) . \\
= & \lambda_{1}+\sum_{n=2}^{\infty} \lambda_{n}=1 .
\end{aligned}
$$

Also we see that

$$
\begin{aligned}
& \sum_{n=2}^{\infty} \frac{\lambda_{n}(1-\alpha)}{(n-\alpha)-(1-\alpha)((1-\lambda)+n \lambda) z_{0}^{n-1}}\left[\frac{(n-\alpha)-(1-\alpha)((1-\lambda)+n \lambda) z_{0}^{n-1}}{1-\alpha}\right] \\
= & \sum_{n=2}^{\infty} \lambda_{n}=1-\lambda_{1} \leq 1 .
\end{aligned}
$$

Therefore from Theorem $1, f(z) \in S_{\lambda}^{*}\left(\alpha, z_{0}\right)$.

Conversely, if $f(z) \in S_{\lambda}^{*}\left(\alpha, z_{0}\right)$, then

$$
a_{n} \leq(1-\alpha) /\left[(n-\alpha)-(1-\alpha)((1-\lambda)+n \lambda) z_{0}^{n-1}\right] \quad(n=2,3, \ldots) .
$$

Set

$$
\lambda_{n}=\left\{\left[(n-\alpha)-(1-\alpha)((1-\lambda)+n \lambda) z_{0}^{n-1}\right] /(1-\alpha)\right\} a_{n} \quad(n=2,3, \ldots)
$$

and 


$$
\lambda_{1}=1-\sum_{n=2}^{\infty} \lambda_{n}
$$

Then $f(z)=\sum_{n=1}^{\infty} \lambda_{n} f_{n}(z)$.

Similarly, the coefficients bounds on $K_{\lambda}\left(\alpha, z_{0}\right)$ enable us to prove

Theorem 7. The extreme points of $K_{\lambda}\left(\alpha, z_{0}\right)$ are given by $f_{1}(z)=z$ and $f_{n}(z)=\frac{n(n-\alpha) z-(1-\alpha) z^{n}}{n(n-\alpha)-(1-\alpha)((1-\lambda)+n \lambda) z_{0}^{n-1}} \quad(n=2,3, \ldots)$.

\section{Convex Families}

Suppose $B$ is nonempty subset of the real interval $(0,1)$, we define $S_{\lambda}^{*}(\alpha, B)$ by

$$
S_{\lambda}^{*}(\alpha, B)=\bigcup_{z_{\gamma} \in B} S_{\lambda}^{*}\left(\alpha, z_{\gamma}\right)
$$

If $\mathbb{B}$ consists of a single element say $z_{0}$ then $S_{\lambda}^{*}\left(\alpha, z_{0}\right)$ is a convex family. Because if $f_{1}(z)$ and $f_{2}(z)$ are in $S_{\lambda}^{*}\left(\alpha, z_{0}\right)$, then it can be seen that for $0 \leq \delta \leq 1$, $f(z)=\delta f_{1}(z)+(1-\delta) f_{2}(z)$ is in $S_{\lambda}^{*}\left(\alpha, z_{0}\right)$. Next we examine this class for other subsets of $B$. We need the following.

Lemma. If $f(z) \in S_{\lambda}^{*}\left(\alpha, z_{0}\right) \cap S_{\lambda}^{*}\left(\alpha, z_{1}\right)$, where $z_{0}$ and $z_{1}$ are distinct positive numbers then $f(z)=z$.

$$
\text { Proof. Taking } \begin{aligned}
f(z) & =a_{1} z-\sum_{n=2}^{\infty} a_{n} z^{n} \quad\left(a_{n} \geq 0\right), \text { we have } \\
a_{1} & =1+\sum_{n=2}^{\infty} a_{n}[(1-\lambda)+n \lambda] z_{0}^{n-1} \\
& =1+\sum_{n=2}^{\infty} a_{n}[(1-\lambda)+n \lambda] z_{1}^{n-1}
\end{aligned}
$$

That is

$$
a_{n}[(1-\lambda)+n \lambda]\left[z_{1}^{n-1}-z_{0}^{n-1}\right]=0 .
$$

Hence $a_{n} \equiv 0$ for $n \geq 2$, and the result follows. 
Theorem 8. If $B$ is contained in the interval $(0,1)$ and $0 \leq \alpha<1$, then $S_{\lambda}^{*}(\alpha, B)$ is a convex family if and only if $B$ is connected.

Proof. Let $B$ be connected. Suppose $z_{0}, z_{1} \in B$ with $z_{0} \leq z_{1}$. If $f(z)=$ $a_{1} z-\sum_{n=2}^{\infty} a_{n} z^{n}$ is in $S_{\lambda}^{*}\left(\alpha, z_{0}\right)$ and $g(z)=b_{1} z-\sum_{n=2}^{\infty} b_{n} z^{n}$ is in $S_{\lambda}^{*}\left(\alpha, z_{1}\right)$ then for $0 \leq \delta \leq 1$, we shall prove that there exists a $z_{2}\left(z_{0} \leq z_{2} \leq z_{1}\right)$ such that $h(z)=\delta f(z)+(1-\delta) g(z)$ is in $S_{\lambda}^{*}\left(\alpha, z_{2}\right)$. Set

$$
\begin{aligned}
t(z)= & {[(1-\lambda) h(z) / z]+\lambda h^{\prime}(z) } \\
=\delta\{ & \left.a_{1}-\sum_{n=2}^{\infty} a_{n} z^{n-1}((1-\lambda)+n \lambda)\right\} \\
& +(1-\delta)\left\{b_{1}-\sum_{n=2}^{\infty} b_{n} z^{n-1}((1-\lambda)+n \lambda)\right\} \\
t(z)=1+ & \delta \sum_{n=2}^{\infty} a_{n}\{(1-\lambda)+n \lambda\}\left(z_{0}^{n-1}-z^{n-1}\right) \\
& +(1-\delta) \sum_{n=2}^{\infty} b_{n}\{(1-\lambda)+n \lambda\}\left(z_{1}^{n-1}-z^{n-1}\right)
\end{aligned}
$$

and we observe that $t(z)$ is real when $z$ is real with $t\left(z_{0}\right) \geq 1$ and $t\left(z_{1}\right) \leq 1$. Hence for some $z_{2}, z_{0} \leq z_{2} \leq z_{1}$, we have $t\left(z_{2}\right)=1$. Since $z_{1}, z_{2}$ and $\delta$ are arbitrary, the family $S_{\lambda}^{*}(\alpha, B)$ is convex.

Conversely, suppose $B$ is not connected. Then we can take $z_{0}, z_{1} \in B$, $z_{2} \notin B$ such that $z_{0}<z_{2}<z_{1}$. Let us assume $f(z)$ and $g(z)$ are not both identity function. Then using (5) and fixing $z=z_{2}$ and allow $\delta$ to vary,

$$
\begin{aligned}
t(\delta)= & t\left(z_{2}, \delta\right) \\
=1 & +\delta \sum_{n=2}^{\infty} a_{n}[(1-\lambda)+n \lambda]\left(z_{0}^{n-1}-z_{2}^{n-1}\right) \\
& \quad+(1-\delta) \sum_{n=2}^{\infty} b_{n}[(1-\lambda)+n \lambda]\left(z_{1}^{n-1}-z_{2}^{n-1}\right) .
\end{aligned}
$$

Since $t\left(z_{2}, 0\right)>1$ and $t\left(z_{2}, 1\right)<1$, there must exists a $\delta_{0}, 0<\delta_{0}<1$, for which $t\left(z_{2}, \delta_{0}\right)=1$. Hence $h(z) \in S_{\lambda}^{*}\left(\alpha, z_{2}\right)$ for $\delta=\delta_{0}$. Since $z_{2} \notin B$ from the 
above lemma it follows that $h(z) \notin S_{\lambda}^{*}(\alpha, B)$. Therefore $S_{\lambda}^{*}(\alpha, B)$ is not a convex family.

\section{References}

[1] H. Silverman, "Univalent functions with negative coefficients", Proc. Amer. Math. Soc. 51 (1975), 109-116.

[2] H. Silverman, "Extreme points of univalent functions with two fixed points", Trans. Amer. Math. Soc. 219 (1976), 387-395.

Department of Mathematics, Karnatak University, Dharwad-580003, India. 